ISSN: 2602-8506

Vol. 3, $\mathrm{N}^{\circ}$ 2, p. 5-25, abril - junio, 2019

\title{
Análisis de la metodología de la ANT para fijar la tarifa de transporte modalidad taxi
}

\section{Analysis of the methodology of the ant to set the transport fee modality taxi}

\author{
Gustavo Javier Aguilar Miranda. ${ }^{1} \&$ José Luis Llamuca Llamuca. ${ }^{2}$
}

DOI: https://doi.org/10.33262/visionariodigital.v3i2.391

\section{Resumen.}

En este artículo se presenta el análisis de la metodología de la ANT para determinar la tarifa de transporte modalidad taxi, y a la vez propone un modelo considerando nuevas variables como distancia, tiempo en vacío y el pago del seguro social del conductor que la metodología vigente no determina, y que influyen significativamente en el cálculo erróneo de los costos y por lo tanto de la tarifa. La investigación de campo se realizó a través de encuestas tanto a usuarios como oferentes del servicio además se obtuvo fichas de observación del monitoreo a los recorridos de un grupo de unidades mediante el acompañamiento en su jornada diaria de trabajo, se realizó un análisis técnico de los costos operacionales y de la oferta de kilómetros que permitió sincerar una tarifa acorde a la realidad local. Los resultados fijaron una tarifa de $\$ 1.38$, el costo por $\mathrm{km}$ de $\$ 0.31$ y un valor de arrancada de $\$ 0.42$, se recomienda una revisión periódica de la tarifa fundamentada en la economía actual.

Palabras claves: sistema de transporte, transporte comercial, tarifa de taxi, metodología.

\footnotetext{
1 Escuela Superior Politécnica de Chimborazo, Facultad de Administración de Empresas, Riobamba, Ecuador, gustavo.aguilar@espoch.edu.ec

${ }^{2}$ Escuela Superior Politécnica de Chimborazo, Facultad de Administración de Empresas, Riobamba, Ecuador, jose.1lamuca@espoch.edu.ec
} 


\section{Abstract.}

This article presents the analysis of the methodology of the ANT to determine the taxi fare, and at the same time proposes a model considering new variables such as distance, idle time and the driver's social security payment that the current methodology does not determines, and that they influence significantly in the erroneous calculation of the costs and therefore of the tariff. The field research was conducted through surveys to both users and service providers, and monitoring records were obtained for the tours of a group of units by means of accompaniment in their daily work, a technical analysis of the operational costs and the offer of kilometers that allowed to open a rate according to the local reality. The results set a rate of $\$ 1.38$, the cost per $\mathrm{km}$ of $\$ 0.31$ and a start value of $\$$ 0.42 , it is recommended a periodic review of the rate based on the current economy

Keywords: Transport system, commercial transport, taxi rate, methodology.

\section{Introducción.}

Para una ciudad constituye un factor imprescindible la regulación de las tarifas del servicio de taxi ofertadas por las diferentes operadoras, cuyo propósito es garantizar una retribución económica, y además, proporcionar a los usuarios un servicio de calidad. Los principales beneficiarios de la presente investigación de forma directa e indirecta serán la población, las operadoras de taxi y los GADs.

En tal sentido, el transporte público es un servicio fundamental y de gran importancia para la movilidad de la población, es el medio a través del cual las personas pueden conectarse de un lugar a otro; sin embargo el crecimiento del servicio de taxi en el país se ha realizado en forma descontrolada y desordenada, para el efecto, la Agencia Nacional de Tránsito (ANT) en uso de sus facultades y con el objetivo de garantizar la prestación de un servicio de calidad en el traslado de las personas y bienes ofrecido por las operadoras a sus usuarios y determinar tarifas socialmente justas, establece una metodología para la fijación de las tarifas, que será tomada como un cálculo referencial y que permitirá a los Gobiernos Autónomos Descentralizados (GADS) regular el cobro monetario por el servicio, esta metodología que en su inicio aplicaba para la modalidad en Taxi Convencional.

Al respecto, el estudio Diseño de un modelo de costos alternativo para la determinación de la tarifa de taxi modalidad convencional en la ciudad de Cuenca realizado por (Aguirre, 2015), afirma que, la metodología de fijación de tarifas de taxis vigente adolece de varias inconsistencias como: la contradicción de las fórmulas para determinar el valor de arranque, desconoce la realidad ecuatoriana al aplicar la normativa Euro 4 recomendada para motores a diésel en donde la mayoría de vehículos utilizados para este fin son a gasolina, el cálculo de retorno de la inversión no se utiliza en ninguna fórmula, en la 
práctica considera difícil o imposible la medición de carreras cortas medianas y largas. Manifiesta además que se desfigura el intento de calcular los costos incurridos de manera objetiva, por lo que considerara necesario plantear un modelo que a más de técnico también sea justo y social.

Por tal razón, es conveniente destacar que los modelos matemáticos que conforman la metodología vigente no consideran las variables, distancia y tiempo en vacío es decir el tiempo de viaje que el taxi utiliza desde su estación hasta el momento de recoger al pasajero y luego de efectuado el servicio de traslado del pasajero, el tiempo que utiliza hasta llegar a su destino.

Este antecedente podría haber generado inconsistencias y perjudicar a los oferentes del servicio de transporte comercial en taxi ya que no se han tomado en cuenta variables que inciden de forma crucial en el cálculo de la tarifa y por ende perjudican en el aspecto económico además de poner en riesgo la calidad del servicio; es así como, el presente estudio, "Análisis de la metodología de la ANT para fijar la tarifa de transporte modalidad taxi”, aportará con lineamientos que permitirán establecer parámetros para fijar una tarifa justa y adecuada que deben pagar los usuarios por el uso de este servicio público y de igual manera que sea rentable para los dueños de las operadoras del servicio de transporte terrestre comercial en taxi, incluyendo variables de tiempo y distancia.

\section{Desarrollo}

\section{Marco teórico referencial}

Se destaca un artículo de la Revista de Obras Públicas, Transportes y Ordenación Territorial de Sevilla en España titulado "Análisis del Sector del Taxi en Andalucía. Contextualización en España y Europa, en el que se realiza una revisión y análisis del sector del taxi en varias ciudades de Europa respecto de su normativa, organización, operativa de solicitud de servicio, sistemas tarifarios y servicios de taxi adaptado, con el fin de mejorar y garantizar el servicio, considerando la equidad entre calidad y rentabilidad. Los sistemas tarifarios establecen tarifas de referencia, tarifas obligatorias en cuanto a los tráficos de corto recorrido. (Maeso, E. González, G. Caballero, J., 2010).

El análisis de los sistemas tarifarios se estructura basándose en cuatro parámetros: servicio mínimo, bajada de bandera, kilómetro recorrido y hora de espera. En ciudades como Londres, se incluyen aspectos como la velocidad y el destino. En Berlín y Roma además de las tarifas generales se dispone de tarifas especiales reducidas, para carreras inferiores a 2 Km. Se diferencian periodos como: laborables diurnos (06:00 a 22:00), laborables nocturnos y festivos, casi todas las ciudades aplican la tarifa diurna. La jornada de trabajo diario se limita a 11 horas con dos descansos de 3 horas máximo y media hora como mínimo. Maeso et al. (2010). 
El artículo "Modelo para el cálculo de la tarifa en equipos de transporte" propone un patrón sencillo para evaluar la estructura de los costos de los equipos empleados para el transporte de bienes y/o personas, brinda una descripción de las variables que inciden en la determinación de las tarifas. Destaca la importancia de obtener una información confiable acerca de las características operacionales de los vehículos para lograr una acertada evaluación de los costos. (Cantillo, 1999).

Un trabajo significativo es el de Juan Aguirre, en su tesis "Diseño de un modelo de Costos alternativo para la determinación de la tarifa de taxi modalidad convencional en la ciudad de Cuenca para el año 2014”, menciona que, los modelos matemáticos legalmente aprobados no consideran en su cálculo las necesidades y deseos de los consumidores, por lo que cree necesario establecer un modelo alternativo de fijación de tarifas de taxis considerando las variables sociales que permiten establecer los umbrales máximos y mínimos de disposición al pago por este servicio, acordes a la realidad socioeconómica de los usuarios de la localidad. (Aguirre, 2015).

\section{Concepto de Transporte}

Existen varias definiciones de transporte, cada una de estas definiciones, aporta una parte importante a su concepto global, entre las que se recalca la de (Garrido, 2001) quien define el transporte como "un sistema organizacional y tecnológico que apunta a trasladar personas y mercancías de un lugar a otro para balancear el desfase espacial y temporal entre los centros de oferta y demanda. Lo anterior plantea el problema de realizar este traslado en forma eficiente y sustentable".

En el mismo sentido, (Izquierdo, 2001) considera al transporte como "un sistema o un subsistema dentro del sistema territorial o incluso del económico, integrado por tres elementos fundamentales interrelacionados entre sí: la infraestructura, el vehículo y la empresa o servicio que viene a constituir la actividad propiamente dicha".

\section{Transporte Terrestre Comercial}

Se denomina servicio de transporte comercial el que se presta a terceras personas a cambio de una contraprestación económica, siempre que no sea servicio de transporte colectivo o masivo. Para operar un servicio comercial de transporte se requerirá de un permiso de operación, Dentro de esta clasificación, entre otros, se encuentran el servicio de transporte escolar e institucional, taxis, tricimotos, carga pesada, carga liviana, mixto, turístico, los cuales serán prestados únicamente por operadoras de transporte terrestre autorizadas para tal objeto. El servicio de taxis se prestará exclusivamente en el área del territorio ecuatoriano, establecido en el permiso de operación respectivo; y, fletado ocasionalmente a cualquier parte del país, estando prohibido establecer rutas y frecuencias. (ANT, 2015) Art. 
57.

\section{Transporte Terrestre Comercial en Taxi}

Consiste en el traslado de terceras personas a cambio de una contraprestación económica desde un lugar a otro dentro del ámbito intracantonal autorizado para su operación, excepcionalmente fuera de ese ámbito cuando sea requerido por el pasajero. Se realizará en vehículos automotores autorizados para ese efecto con capacidad de hasta cinco pasajeros incluido el conductor. Deberán cumplir las exigencias definidas en el reglamento específico emitido para el efecto y las ordenanzas que emitan los GADS. (ANT, 2012) Art. 62.

\section{Subtipos de Transporte Terrestre Comercial en Taxi}

Convencionales: Consiste en el traslado de terceras personas mediante la petición del servicio de manera directa en las vías urbanas, en puntos específicos definidos dentro del mobiliario urbano (paradero de taxi), o mediante la petición a un centro de llamadas.

Ejecutivos: Consiste en el traslado de terceras personas mediante la petición del servicio, exclusivamente, a través de un centro de llamadas, siendo el recorrido autorizado el solicitado por el cliente. (ANT, 2012) Art. 62.

\section{Operadoras de Transporte Terrestre}

Constituye una operadora de transporte terrestre, toda persona jurídica, sea cooperativa o compañía, que habiendo cumplido con todos los requisitos exigidos en esta Ley, su Reglamento y demás normativa aplicable, haya obtenido legalmente el título habilitante para prestar el servicio de transporte terrestre en cualquiera de sus clases y tipos. (ANT, 2015) Art. 77.

\section{Rutas y Frecuencias}

Se entenderá por ruta o línea de servicio de transporte público al trazado o conjunto de vías sobre las que se desplazan los vehículos para otorgar el servicio, atendidos por una misma operadora. (ANT, 2012) Art. 110.

\section{Metodologia.}

La metodología de trabajo aplicada en la presente investigación se basa principalmente en el análisis de la metodología aprobada por la ANT, y estudios previos que determinaron observaciones a esta metodología, con la aplicación de medidas estadísticas que permitan identificar, determinar, promediar, agrupar y condensar la información en parámetros o 
estimadores a todas las variables que conforman los insumos para la determinación de la tarifa en esta modalidad de servicio.

Para ello se estableció las siguientes etapas:

- Recopilación de datos en campo e información primaria

- Tabulación y procesamiento de la información

- Análisis de la Metodología de la Agencia Nacional de Tránsito

- Determinación y cálculos de variables involucradas

- Cálculo de las tarifas

Cada una de estas etapas contempló más de dos actividades específicas que permiten obtener ya sea una tendencia o la información respectiva de una o más variables incidentes en la investigación realizada.

El tamaño de muestra de acuerdo a las recomendaciones nacionales e internacionales (MTOP, 2010) se determinó a partir de la siguiente ecuación:

$$
\boldsymbol{n}=\frac{Z^{2} * N * p * q}{E^{2}(N-1)+Z^{2} * p * q}
$$

La muestra calculada con los datos correspondientes a la población del cantón de Latacunga de acuerdo a la fórmula aplicada fue de 383 encuestas (pero se aplicó un total de 385 encuestas). Por lo que el desarrollo de la recopilación de datos se dividió en tres actividades específicas que se detallan a continuación:

\section{Determinación de la demanda modal}

Esta actividad consistió en la aplicación de encuestas origen destino de intercepción a los usuarios en general que están en capacidad de realizar un viaje por si mismos; por medio de la cual fue posible cuantificar la demanda actual determinada por el número de viajes entre zonas según el medio de transporte utilizado.

\section{Determinación de la oferta del servicio.}

La determinación de la oferta de servicio no es más que la identificación del número de kilómetros recorridos por el vehículo para un número de carreras realizadas por éste considerando el porcentaje de no ocupación. 
La actividad requirió de la aplicación de un formulario de monitoreo o seguimiento periódico (cada dos horas) a tres unidades vehiculares de diferente marca por cada operadora, durante la jornada laboral en cuatro días típicos y un día atípico como lo establece la metodología técnica para este tipo de estudios; con el fin de cuantificar las carreras cortas, carreras intermedias y carreras largas con sus respectivas distancias recorridas en kilómetros para cada tipo de vehículo. Para ello se validó la información a través de los tickets o facturas emitidas en cada carrera realizada.

Obtención de información de costos operacionales.

Completando la recolección de datos de campo o información primaria se aplicó un tercer formulario diseñado para identificar los diferentes costos y gastos en los que incurre cada propietario de este tipo de automotor durante la prestación del servicio. Para ello se entrevistó a una muestra representativa de propietarios de vehículos de cada operadora según el tamaño de la flota vehicular existente $70 \%$ del total de la flota vehicular según las recomendaciones del manual de estudios de transporte urbano y para garantizar resultados más confiables).

\section{Resultades.}

\section{Sistema de transporte}

El servicio de transporte terrestre en el cantón se encuentra en un estado de servicio mejorable, relativamente bueno y suficiente para el transporte terrestre urbano intracantonal. (Unidad de Movilidad Latacunga, 2019). La estructura del sistema de transporte terrestre urbano está distribuida en 5 modalidades, detallados en la tabla 1. La flota vehicular para el transporte de pasajeros es de 1052 unidades representando un indicador de 193 habitantes por unidad.

Tabla 1: Sistema de transporte terrestre Latacunga

\begin{tabular}{|l|c|c|}
\hline OPERADORA DE TRANSPORTE & $\begin{array}{c}\text { NÚMERO DE OPERADORAS } \\
\text { CANTON LATACUNGA }\end{array}$ & $\begin{array}{c}\text { NUMERO DE } \\
\text { VEHÍCULOS }\end{array}$ \\
\hline Transporte comercial, carga liviana & 27 & 235 \\
\hline Transporte comercial, en taxi ejecutivo & 13 & 225 \\
\hline Transporte comercial, en taxi convencional & 35 & 348 \\
\hline Transporte comercial escolar e institucional & 11 & 145 \\
\hline Transporte público urbano & 2 & 99 \\
\hline \multicolumn{1}{|c|}{ TOTAL } & & $\mathbf{1 0 5 2}$ \\
\hline
\end{tabular}

Fuente: Unidad de movilidad Latacunga (2019)

Realizado por: Los autores 
Operadoras de transporte comercial en taxi

De acuerdo la información proporcionada por la UMTTTSVL, la oferta de transporte en la modalidad de Taxi en la ciudad de Guano, está conformada por treinta y cinco (35) operadoras de Transporte Comercial de Pasajeros en Taxi Convencional y trece (13) operadoras de transporte Comercial en Taxi Ejecutivo, conformadas por una flota vehicular de trescientos cuarenta y ocho (348) y doscientos veinte y cinco (225) unidades respectivamente, las mismas se encuentran debidamente registradas y legalizadas en la institución.

\section{Partición Modal}

Gráfico 1. Partición modal.

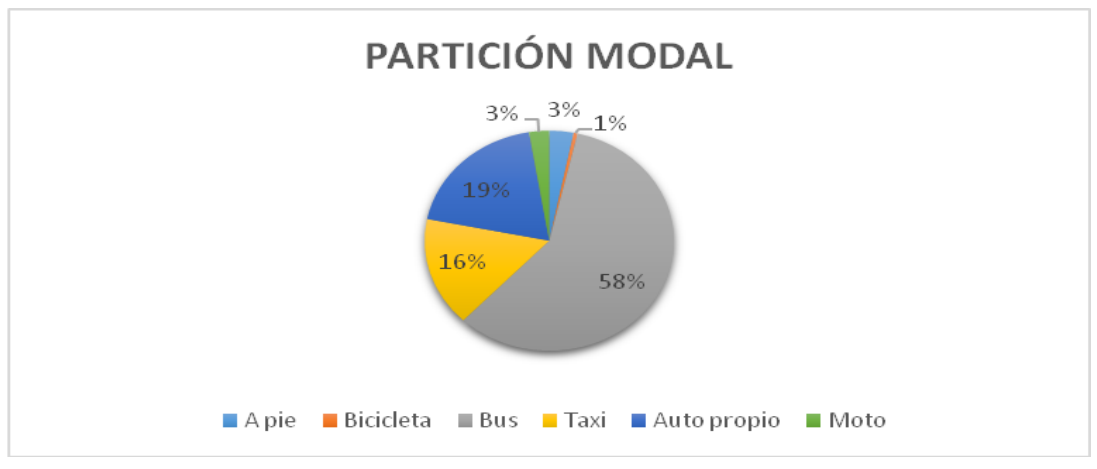

Fuente: Elaboración propia

Análisis: Del total de encuestados el 58\% utiliza como medio transporte el bus, seguido del $19 \%$ que utiliza auto propio, el 16\% utiliza como medio de transporte el taxi, el $3 \%$ utiliza moto, el $3 \%$ se moviliza a pie y, el medio de transporte menos utilizado es la bicicleta con el $1 \%$.

Frecuencia de uso

Gráfico 2. Frecuencia de uso

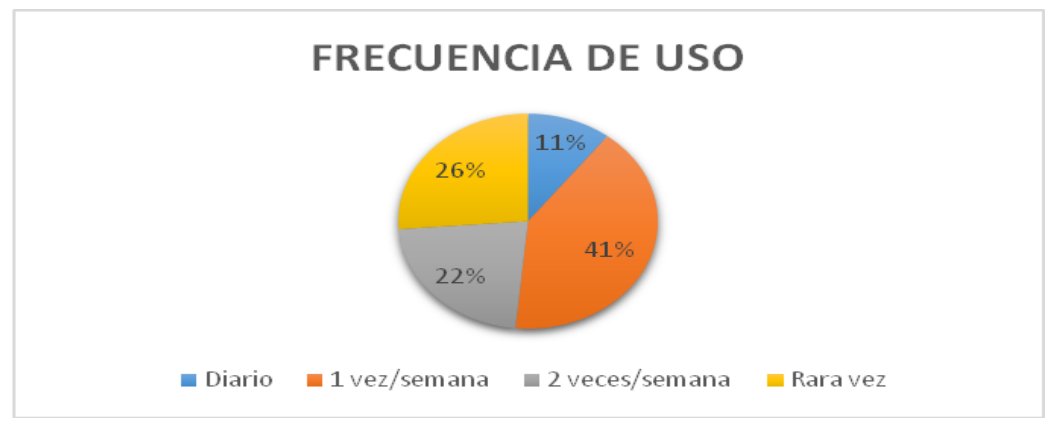

Fuente: Elaboración propia 
Vol. 3, $\mathrm{N}^{\circ}$ 2, p. 5-25, abril - junio, 2019

Análisis: Los resultados demuestran que el $41 \%$ de los encuestados utilizan el servicio de taxi 1 vez por semana, el $26 \%$ indican que usan el servicio rara vez, seguido del $22 \%$ que lo utiliza dos o más veces por semana, y el $11 \%$ utiliza el servicio diariamente.

Horario de uso

Gráfico 3. Horario de uso

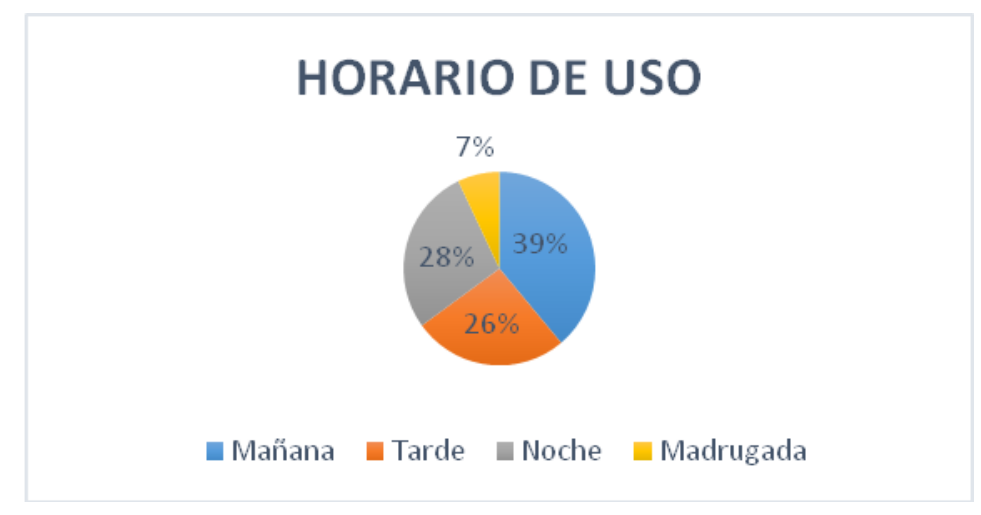

Fuente: Elaboración propia

Análisis: El 39\% de los encuestados utiliza el servicio de taxi en la mañana, mientras que el $28 \%$ lo utiliza en la noche, el $26 \%$ en la tarde y el $7 \%$ utiliza el servicio en la madrugada.

\section{Oferta del servicio}

Para el estudio de la oferta del servicio se aplicó una encuesta al universo de unidades de las compañías que operan en el cantón distribuidas en distintas parroquias, conformado por 573 taxis, 348 convencionales y 225 ejecutivos.

\section{Conductores con afiliación al IESS}

Gráfico 4. Conductores con afiliación al IESS

\section{CONDUCTORES CON AFILIACIÓN AL IESS}

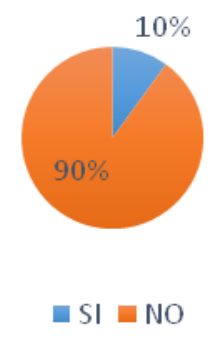

Fuente: Elaboración propia 
Análisis: Del total de encuestados el $90 \%$ no posee afiliación al IESS y apenas el 10\% de los encuestados cuenta con este beneficio.

Número de Carreras Cortas, Intermedias y Largas.

Gráfico 5. Número de carreras cortas intermedias y largas

\section{NÚMERO DE CARRERAS}

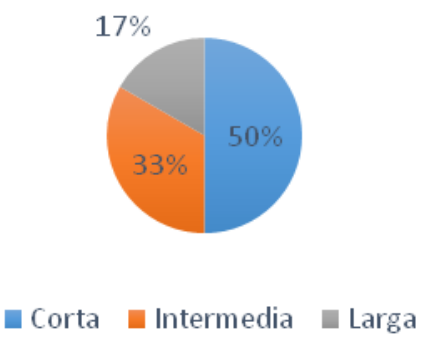

Fuente: Elaboración propia

Análisis: El promedio de carreras realizadas al día es de 36, de las cuales, el 50\% corresponden a carreras cortas, el $33 \%$ a carreras intermedias y el $17 \%$ a las carreras largas

\section{Tiempo en vacío}

Cabe destacar que, en la ficha de observación, también se recogió información acerca de la distancia y tiempo en vacío de cada carrera realizada por las distintas unidades, datos que sirvieron para el cálculo de la tarifa, incluyendo así estas variables que no son consideradas en la metodología de la ANT.

Para calcular el tiempo en vacío se tomaron en cuenta los tiempos que la unidad utiliza cuando sale desde la estación a recoger al usuario y el tiempo de regreso a la parada cuando la carrera termina, mediante la sumatoria de los tiempos en vacío se obtuvo como resultado una media de 6 minutos de tiempo promedio en vacío. Este valor se incluye en la Fórmula de Tarifa mínima de carrera, sumado a los kilómetros recorridos con pasajeros.

\section{Distancia en vacío}

Para el cálculo de la distancia en vacío en la ficha de observación se recogió información sobre la distancia en vacío de recogida al usuario y la distancia en vacío de regreso a la parada; de la sumatoria de las dos distancias en vacío se obtuvo una media de $1.36 \mathrm{~km}$ equivalente a la distancia en vacío promedio. Este valor se incluye en la Fórmula de Tarifa 
mínima de carrera, sumado a la variable de número de minuto de espera en carrera realizada.

\section{Análisis de la Metodología de la Agencia Nacional de Tránsito (ANT)}

La ANT como organismo de control establece la metodología de cálculo referencial para las tarifas por la prestación del servicio de transporte terrestre comercial en taxi en el año 2014, Al respecto, el estudio Diseño de un modelo de costos alternativo para la determinación de la tarifa de taxi modalidad convencional en la ciudad de Cuenca realizado por (Aguirre, 2015), afirma que, la metodología de fijación de tarifas de taxis vigente adolece de varias inconsistencias como: la contradicción de las fórmulas para determinar el valor de arranque, desconoce la realidad ecuatoriana al aplicar la normativa Euro 4 recomendada para motores a diésel en donde la mayoría de vehículos utilizados para este fin son a gasolina, el cálculo de retorno de la inversión no se utiliza en ninguna fórmula. Manifiesta además que se desfigura el intento de calcular los costos incurridos de manera objetiva, por lo que considerara necesario plantear un modelo matemático más técnico.

Por tal razón, es conveniente destacar que los modelos matemáticos que conforman la metodología vigente no consideran las variables de distancia y tiempo en vacío. La metodología vigente no especifica el procedimiento para determinar las distancias promedias para carreras cortas, intermedias y largas ni los parámetros a considerar para su cálculo. Para este análisis en el presente estudio se realizó un acompañamiento al recorrido de las unidades en distintos días y horarios y mediante un cálculo promedio de las distancias recorridas por las unidades se pudo determinar estos valores.

Entre otras referencias sobre el análisis de la metodología cabe destacar a (Erráez. G, Orellana M, 2018), quienes manifiestan que una tarifa social debería recoger las necesidades de rentabilidad, recuperación de los costos, mano de obra y demás aspectos de relevancia para los proveedores del servicio, así como la capacidad de pago, ingresos y la frecuencia de uso de los usuarios. De esta manera se cumpliría con el principio de equidad y trato justo.

Es importante señalar que dentro de los costos fijos la metodología de la ANT incluye como rubros dos tipos de seguros, el seguro obligatorio de accidentes de tránsito SPATT que se cancela anualmente junto con el valor de la matriculación vehicular, y el seguro privado anual para el vehículo con cobertura todo riesgo; cómo se puede inferir esta metodología no toma en cuenta el pago al seguro social que según la Constitución de la república en su artículo 367 es un derecho irrenunciable de todas las personas, se rige por los principios del sistema nacional de inclusión y equidad social y por los de obligatoriedad, suficiencia, integración, solidaridad y subsidiaridad. 
Tomado en cuenta el riesgo de trabajo al que expone este tipo de actividad laboral, se debería considerar como una forma de estabilidad económica que cubra al propietario o conductor de la unidad de taxi en el caso de accidentes de trabajo, así como asegurar una pensión mensual mediante la jubilación.

Entre otros aspectos, la metodología de la ANT se refiere al financiamiento que se realiza para la adquisición del vehículo sin tomar en cuenta el costo del puesto en la compañía, además, la fórmula para el cálculo del valor residual del activo, es decir el valor que tendrá el vehículo al final de su vida útil, fue modificada mediante Resolución 107-DIR-2014ANT, si bien es cierto que la misma calcula un valor residual de la recuperación de capital pero no un costo mensual de capital. Además, dentro de sus variables de cálculo se encuentra el Valor de salvamento, el mismo que refiere a la valoración monetaria del Plan Renova, cuyo incentivo económico implantado por el Gobierno Nacional para el proceso de chatarrización de vehículos culminó el 31 de diciembre del 2015; esta iniciativa durante el año 2017 fue promovida por el sector privado.

\section{Propuesta metodológica para el cálculo de la tarifa}

\section{Oferta de Kilómetros}

Para el cálculo de la tarifa se toman en cuenta dos elementos principales como son la oferta de kilómetros y los costos operacionales. En este sentido, la oferta de kilómetro está conformada por información referente al número de días que labora la unidad al mes; el número de carreras y la distancia promedio en $\mathrm{Km}$ recorridos ya sean cortas intermedias y largas; el número de kilómetros que recorre la unidad al día con pasajeros; el número de kilómetros que recorre la unidad al día sin pasajeros.

La información recogida a través de encuestas permitió determinar los datos promedio para el cálculo de la oferta de kilómetros.

Tabla 2: Oferta de Kilómetros

\begin{tabular}{|l|c|}
\hline \multicolumn{1}{|c|}{ Etiquetas } & Datos \\
\hline \# de días promedio que labora la unidad al mes & 26 \\
\hline \# de Carreras Cortas (CC) & 18 \\
\hline Distancia Promedio en km recorridos CC & 1.5 \\
\hline \# de Carreras Intermedias (CI) & 12 \\
\hline Distancia Promedio en km recorridos CI & 3.5 \\
\hline \# de Carreras Largas (CL) & 6 \\
\hline Distancia Promedio en km recorridos CL & 9 \\
\hline
\end{tabular}


Vol. 3, $\mathrm{N}^{\circ}$ 2, p. 5-25, abril - junio, 2019

\begin{tabular}{|l|c|} 
Total de carreras promedio realizadas al día & 36 \\
\hline Km recorridos al día por la unidad con pasajeros & 123 \\
\hline Km recorridos al día por la unidad sin pasajeros & 49 \\
\hline \% de NO ocupación & $28 \%$ \\
\hline Km recorridos / día & 172 \\
\hline Km recorridos / mes & 4472 \\
\hline Km recorridos / año & 53664 \\
\hline
\end{tabular}

Fuente: Elaboración propia

Fórmulas de cálculo:

$$
\begin{gathered}
\text { Kdía }=\sum(N C C \times K m C C)+(N C I \times K m C I)+(N C L \times K m C L) \\
\text { Kdía }=172
\end{gathered}
$$

$$
\begin{gathered}
\text { Kmes }=\left[\sum(N C C \times \text { KmCC })+(N C I x \text { KmCI })+(N C L \times \text { KmCL })\right] \times \text { Dlab } \\
\text { Kmes }=4472 \\
\text { NC }=\sum(N C C+N C I+N C L) \\
N C=53664 \\
\% \text { NO }=\frac{\text { Krsp }}{\sum(\text { Krsp }+ \text { Krcp })} \times 100 \\
\% \mathrm{NO}=28 \%
\end{gathered}
$$

Costos operacionales

Costos fijos

$$
\text { Fórmula de cálculo: } \quad C f i=\sum(M O+S e g+L e g+G A+G O P)
$$

Tabla 3: Costos Fijos

\begin{tabular}{|l|c|c|}
\hline \multicolumn{1}{|c|}{ Etiquetas } & Costo / Año & Costo / Mes \\
\hline Mano de obra & 7021.8 & 585.15 \\
\hline Seguro Social & 782.88 & 65.24 \\
\hline Legalización & & \\
\hline Matriculación vehicular & 51 & 4.25 \\
\hline SPATT & 48 & 4.00 \\
\hline Permiso de operación & 41.8 & 3.48 \\
\hline
\end{tabular}


ISSN: 2602-8506

www.visionariodigital.org

Vol. 3, N², p. 5-25, abril - junio, 2019

\begin{tabular}{|c|c|c|}
\hline Revisión vehicular & 18.09 & 1.52 \\
\hline Impuesto al rodaje municipal y provincial & 22 & 1.83 \\
\hline \multicolumn{3}{|l|}{ Gastos administrativos } \\
\hline Cuotas sociales (incluye: comunicación/ radio, garaje) & 428.16 & 35,68 \\
\hline \multicolumn{3}{|l|}{ Gastos operativos } \\
\hline Kit de seguridad & 40 & 3.33 \\
\hline Taxímetro & 52.56 & 4.38 \\
\hline Total & 856.29 & 708.87 \\
\hline
\end{tabular}

Fuente: Elaboración propia

$$
C f i=\sum(M O+S e g+L e g+G A+G O P)
$$

Cfi $=708.87$ mensual carrera diurna $\quad$ Cfi $=855.15$ mensual carrera nocturna

Para el cálculo de la tarifa nocturna se incluye al valor de la remuneración de 585.15 de mano de obra el $25 \%$ de incremento estipulado en el código de trabajo.

Costos variables

$$
\text { Fórmula de cálculo: } \quad \mathrm{Cvi}=\sum(\mathrm{Com}+\mathrm{Rod}+\mathrm{Mpre}+\mathrm{Mco})
$$

Com: Combustible, según la metodología de la ANT el costo del combustible dependerá de la potencia del motor, de las condiciones de trabajo y del valor unitario del combustible. ANT (2014). En este caso, las mediciones del rendimiento del consumo de combustible se realizaron con relación al vehículo promedio Chevrolet Aveo Family cilindraje 1.5, como se evidencia en la Tabla 4, se considera el factor de consumo correspondiente al rango de 13.1 a 130, en vista de que el RLOTTTSV establece como límites máximos y rangos moderados de velocidad vehicular permitidos en las vías públicas de $50 \mathrm{~km} / \mathrm{h}$.

Tabla 4: Funciones de los factores de consumo de combustible de vehículos ligeros

\begin{tabular}{|c|c|c|}
\hline \multirow{2}{*}{$\begin{array}{l}\text { CILINDRADA } \\
\text { (L) }\end{array}$} & $\begin{array}{c}\text { RANGO DE } \\
\text { VELOCIDADES } \\
\end{array}$ & \multirow{2}{*}{$\begin{array}{l}\text { FACTOR DE CONSUMO } \\
(\mathbf{g} / \mathbf{k m})\end{array}$} \\
\hline & (km/hora) & \\
\hline \multirow{2}{*}{$1.4<\mathrm{L}<2.0$} & 5 a 13.1 & $428.06-46.696 \mathrm{~V}+1.697 \mathrm{~V}^{2}$ \\
\hline & 13.1 a 130 & $135.44-2.314 \mathrm{~V}+0.0144 \mathrm{~V}^{2}$ \\
\hline
\end{tabular}
según cilindrada y velocidad.

Fuente: ANT (2014)

Reemplazando los valores de la tabla 4 tenemos que el factor de consumo es igual a 55.74. 
Para obtener el gasto promedio de consumo de combustible es necesario el costo de combustible comercial, los resultados de las encuestas determinaros que el combustible que usa el total de encuestados es gasolina extra, el precio de este tipo de combustible tuvo una variación a partir del mes de diciembre del 2018 , de $\$ 1.48$ se incrementó a $\$ 1.85$ debido a la rebaja del subsidio; además los datos de la tabla 2. de la oferta de kilómetros: km recorridos / día $=172 ; \mathrm{km}$ recorridos $/$ mes $=4472 ;$ y el valor de factor de consumo.

Gasto promedio de combustible $=(\mathrm{km}$ recorridos $*$ precio combustible $) /$ factor de consumo

Gasto promedio de combustible diario $=\mathbf{5 . 7}$

Gasto promedio de combustible mensual $=\mathbf{1 4 8 . 6 4}$

Rod: Para el cálculo de Rodamiento se recogió información mediante proformas de distintas casas comerciales, en vista de que los resultados de las encuestas indicaron que el $47.9 \%$ de los encuestados usan neumáticos extranjeros y el $52.1 \%$ utiliza neumáticos nacionales.

Tabla 5: Costo en neumáticos.

\begin{tabular}{|c|c|c|c|}
\hline $\begin{array}{c}\text { Promedio de \# } \\
\text { neumáticos/año }\end{array}$ & $\begin{array}{c}\text { Costo Unitario / } \\
\text { neumático }\end{array}$ & $\begin{array}{c}\text { Costo neumáticos / } \\
\text { mes }\end{array}$ & $\begin{array}{c}\text { Costo neumáticos / } \\
\text { año }\end{array}$ \\
\hline 5.6 & 88.57 & 41.05 & 492.60 \\
\hline
\end{tabular}

Fuente: Elaboración propia

El mantenimiento se calcula tomando en cuenta el kilometraje recorrido por las unidades, en base a los datos de la tabla 2 de oferta de kilómetros se obtiene los datos de los kilómetros recorridos por mes 4472 y los kilómetros recorridos por año 53664.

Mpre: El Mantenimiento preventivo

Tabla 6: Rubros Mantenimiento Preventivo

\begin{tabular}{|l|c|c|c|}
\hline \multicolumn{1}{|c|}{ Rubros } & $\begin{array}{c}\text { Estimado de } \\
\text { cambio (Km) }\end{array}$ & Costo/cambio & Costo/mes \\
\hline $\begin{array}{l}\text { Aceite y lubricante de motor } \\
\text { (gal)/mes }\end{array}$ & 5000 & 27.53 & 32.80 \\
\hline $\begin{array}{l}\text { Aceite y lubricante caja } \\
\text { (1)/mes }\end{array}$ & 60000 & 57,89 & 5.13 \\
\hline Aceite hidráulico (1)/mes & 30000 & 25,41 & 4.51 \\
\hline Filtro de aceite & 50000 & 8.72 & 15.62 \\
\hline
\end{tabular}


ISSN: 2602-8506

www.visionariodigital.org

Vol. 3, N², p. 5-25, abril - junio, 2019

\begin{tabular}{|l|c|c|c|}
\hline Filtro de aire & 10000 & 8.56 & 14.98 \\
\hline Filtro de combustible & 15000 & 7.02 & 13.90 \\
\hline Engrase general g/año & 50000 & 27,05 & 3.42 \\
\hline Kit de embrague & 90000 & 184.80 & 9.19 \\
\hline Betería /mes & 50000 & 125,44 & 10.45 \\
\hline Alineación y balanceo/mes & 10000 & 22,85 & 10.23 \\
\hline Frenos (pastillas, zapatas)/mes & 10000 & 56 & 25.10 \\
\hline $\begin{array}{l}\text { Bandas de accesorios y de } \\
\text { distribución/mes }\end{array}$ & 65000 & 42,58 & 2.93 \\
\hline Limpieza de inyectores & 30000 & 30,58 & 4.56 \\
\hline $\begin{array}{l}\text { Amortiguadores, terminales de } \\
\text { dirección, bujes de suspensión }\end{array}$ & 60000 & 334.57 & 24.97 \\
\hline $\begin{array}{l}\text { Rectificación de disco de } \\
\text { freno y tambor }\end{array}$ & 30000 & 39,11 & 5.84 \\
\hline Lavado motor/carrocería & 5000 & 13,44 & 13.44 \\
\hline \multicolumn{1}{|c|}{ TOTAL } & $\mathbf{1 0 1 1 . 5 5}$ & $\mathbf{1 9 7 . 0 5}$ \\
\hline
\end{tabular}

Fuente: Elaboración propia

Mco: El Mantenimiento correctivo

Tabla 7: Rubros Mantenimiento Correctivo.

\begin{tabular}{|l|c|c|c|}
\hline \multicolumn{1}{|c|}{ Rubros } & $\begin{array}{c}\text { Estimado de } \\
\text { cambio (Km) }\end{array}$ & Costo/cambio & $\begin{array}{c}\text { Costo } \\
\text { /mes }\end{array}$ \\
\hline Reemplazo bomba de inyección & 200000 & 325,75 & 7.29 \\
\hline $\begin{array}{l}\text { Reemplazo elementos sistema de } \\
\text { suspensión }\end{array}$ & 100000 & 352.06 & 15.77 \\
\hline Elementos sistema eléctrico & 15000 & 44.35 & 13.24 \\
\hline Reparación del motor & 250000 & 1349.60 & 24.17 \\
\hline Reparación de caja & 300000 & 1220.93 & 18.22 \\
\hline \multicolumn{1}{|c|}{ TOTAL } & & $\mathbf{3 2 9 2 . 7 0}$ & $\mathbf{7 8 . 7 0}$ \\
\hline
\end{tabular}

Fuente: Elaboración propia

Aplicando la fórmula se obtiene el valor de los costos variables

$$
\begin{gathered}
C v i=\sum(148.64+41.05+197.05+78.70) \\
C v i=\mathbf{4 6 5 . 4 4}
\end{gathered}
$$


Costos de capital

Tabla 10: Costos de capital.

\begin{tabular}{|c|c|c|c|c|c|c|}
\hline CP +D & CP & D & tc & & If & Kd \\
\hline Inversión & $\begin{array}{c}\text { Capital } \\
\text { propio }\end{array}$ & Endeudamiento & $\begin{array}{c}\text { Tasa de } \\
\text { interés real } \\
(\boldsymbol{\%})\end{array}$ & $\begin{array}{c}\text { Plazo } \\
\text { deuda } \\
\text { (años) }\end{array}$ & $\begin{array}{c}\text { Impuesto } \\
\text { fiscal }\end{array}$ & $\begin{array}{c}\text { Interés } \\
\text { deuda }\end{array}$ \\
\hline 21877 & 5323 & 9506 & 0.128 & 4 & 0 & 22343 \\
\hline
\end{tabular}

Fuente: Elaboración propia.

Con los datos obtenidos aplicamos la fórmula:

$$
\mathrm{Cci}=\mathrm{tc} \frac{\mathrm{Cp}}{\mathrm{CP}+\mathrm{D}}+\mathrm{Kd}(1-\text { if }) \frac{\mathrm{D}}{\mathrm{CP}+\mathrm{D}}
$$

$$
\text { Cci }=199.89
$$

Cálculo de la tarifa mínima de carrera en taxi convencional y ejecutivo

Para calcular la tarifa mínima de carrera es necesario calcular el valor de costo por kilómetro recorrido, el valor de la arrancada, el costo minuto de espera y la eficiencia operacional. La información recogida mediante los recorridos con las unidades permitió incluir los valores para las variables consideradas para esta metodología como son la distancia y tiempo en vacío.

De igual manera se recogió la información respecto de los tiempos que existen entre cada carrera, denominado minuto de espera en carrera realizada (Mmecp), el cálculo promedio de los tiempos efectuados de cada carrera refleja un valor de 1 minuto.

Costo por kilómetro recorrido

$$
\begin{gathered}
C k=\frac{\sum(C f i+C v i+C c i)}{K m e s} \\
C k=0.31(\text { diurno }) \quad C k=0.34 \text { (nocturno) }
\end{gathered}
$$

Arrancada

$$
\mathbf{A r}=\frac{\text { Ckx (\%NO x Kdía) }}{\mathrm{NC}}
$$




$$
\text { Ar }=0.42(\text { diurno }) \quad A r=0.46(\text { nocturno })
$$

Costo minuto de espera

$$
\text { Cme }=\frac{K d i a \text { x } C k}{12 h \times 60 \mathrm{~min}}
$$

$$
\text { Cme }=0.07(\text { diurno }) \quad \text { Cme }=0.08 \text { (nocturno) }
$$

Con los resultados obtenidos se efectuó el cálculo de la tarifa mínima de carrera diurna y nocturna, para lo cual se planteó, la siguiente fórmula:

$T M C=\sum(A r+(C k x$ Kmrcp $)+($ Cme $x$ Mmecp $)+($ TvxCme $)$

\section{Tarifa mínima de Carrera Diurna}

$$
\begin{gathered}
\mathrm{TMC}=0,42+(0,31 \times 1,5)+(0,07 \mathrm{x} 1)+(6 \mathrm{x} 0,07) \\
\text { TMC }=\mathbf{1 , 3 8}
\end{gathered}
$$

\section{Tarifa mínima de Carrera Nocturna}

$$
\mathrm{TMC}=0,46+(0,34 \times 1,5)+(0,08 \times 1)+(6 \times 0,08)
$$

$$
\mathrm{TMC}=\mathbf{1 , 5 3}
$$

La información obtenida nos permite determinar un costo de la tarifa mínima de carrera para la jornada diurna de $\$ 1.38$ y para la jornada nocturna de $\$ 1.53$, fundamentada en la metodología establecida por la ANT incluidas las variables de distancia y tiempo en vacío.

\section{Conclusiones.}

- Entre las falencias de mayor relevancia del modelo matemático de la metodología de ANT se puede mencionar la ausencia de variables de tiempo, distancia en vacío y el pago del seguro social del conductor en los costos de operación que inciden en su cálculo, no especifica el procedimiento ni los parámetros para establecer las distancias promedias entre carreras cortas intermedias y largas. 
- El análisis técnico de los costos operacionales y la oferta de kilómetros permitió determinar una tarifa acorde a la realidad del cantón Latacunga con lo que se pretende mejorar la calidad del servicio.

- La propuesta metodológica determinó una tarifa mínima de carrera en el día de $\$ 1.38$, el costo por $\mathrm{km}$ recorrido es de $\$ 0.31$ y la arracada $\$ 0.42$, el promedio de días que labora una unidad es de 26 , el promedio de carreras diarias 36 y el promedio de km que recorre una unidad por día es de 172.

\section{Referencias bibliográficas.}

Agencia Nacional de Tránsito del Ecuador. (2014). Resolución No. 107-dir-2014-ANT. Reforma a la metodología para la fijación de tarifas (Taxi convencional) (pág. 4). Quito: Registro Oficial.

Aguirre, J. (2015). Diseño de un modelo de cotos alterntivo para la determinación de la tarifa de taxi modalidad convencional en la ciudad de Cuenca para el año 2014. Cuenca.

ANT. (2012). Reglamento General para la aplicación a la Ley Orgánica de Transporte Terrestre, Tránsito y Seguridad Vial. Registro Oficial. Obtenido de https://www.obraspublicas.gob.ec/wp-content/uploads/downloads/2015/03/DecretoEjecutivo-No.-1196-de-11-06-2012-REGLAMENTO-A-LA-LEY-DETRANSPORTE-TERRESTRE-TRANSITO-Y-SEGURIDAD-VIA.pdf

ANT. (2014). Resolución No.073-DIR-2014-ANT. Metodología para la fijación de tarifas (Taxi Convencional). Obtenido de https://www.ant.gob.ec/index.php/transito7/resoluciones-2014/file/2396-resolucion-no-073-dir-2014-ant-metodologia-para-lafijacion-de-tarifas-taxi-convencional

ANT. (2015). Ley Orgánica de Transporte Terrestre, Tránsito y Seguridad Vial. Obtenido de https://www.ant.gob.ec/index.php/ant/base-legal/ley-organica-reformatoria-a-laley-organica-de-transporte-terrestre-transito-y-seguridad-vial

Cantillo, V. (1999). Modelo para el cálculo de la tarifa de equios de transporte. . Ingeniería y Desarrollo. Universidad del Norte.

Domínguez, H. (2006). El servicio invisible : fundamento de un buen servicio al cliente. Bogotá: Ecoe Ediciones.

Erráez. G, Orellana M. (2018). Análisis para la determinación de la tarifa de taxi convencional en el cantón Nabón. Cuenca.

GAD Garrido, R. (2001). Modelación de sistemas de distribución de carga. Santiago de Chile: Ediciones Universidad Católica.

Hernández. R, Fernández. C, Baptista. P. (2014). Metodología de la Investigación. México D. F.: McGraw-Hill.

INEC. (2010). Resultados del Censo 2010 de Población y Vivienda en el Ecuador. Obtenido de Instituto Nacional de Estadísticas y Censos: 
http://www.ecuadorencifras.gob.ec/wp-content/descargas/Manu-lateral/Resultadosprovinciales/chimborazo.pdf

INEC. (2016). Encuesta Nacional de Empleo, Desempleo y Subempleo. Indicadores Laborales Marzo 2016. Obtenido de Instituto Nacional de Estadísticas y Censos: http://www.ecuadorencifras.gob.ec/documentos/web-inec/EMPLEO/2016/Marzo2016/Presentacion\%20Empleo_0316.pdf

INEC. (2019). Medidas de Inflaciòn. Indice de precios al consumidor. Obtenido de http://www.ecuadorencifras.gob.ec/documentos/webinec/Inflacion/2018/Diciembre-2018/01\%20ipc\%20Presentacion_IPC_dic2018.pdf

Izquierdo, R. (2001). Transportes un enfoque integral. Tomo I Transporte y Economía del transporte. Madrid: Editorial Ugarte S. I.

Maeso, E. González, G. Caballero, J. (2010). El sector del taxi en Andalucía. Contextualización en España y Europa. Reflexiones. Revista de Obras Públicas, transporte y ordenación territorial, 55-80.

MDMQ. (2009). Municipio del Distrito Metropolitano de Quito. Plan MAestro de Movilidad para el Distrito Metropolitanode Quito. Obtenido de file://C:/Users/C\&DSYSTEMS/Downloads/LFLACSO-01-DMQ.pdf

MDMQ. (2015). Municipio del Disrito Metropolitno de Quito. Obtenido de Gobierno Abierto. Acta Transcrita de la Sesión extraordinaria de la Comisión de Movilidad. 31 de Marzo de 2015

MInisterio de Trabajo. (2019). Salarios Mínimos Sectoriales 2019. Obtenido de http://www.trabajo.gob.ec/biblioteca/

Minsiterio de Trabajo. (2012). Código de Trabajo. Registro Oficial Suplemento 167 de 16dic-2005. Obtenido de http://www.trabajo.gob.ec/wpcontent/uploads/downloads/2012/11/C\%C3\%B3digo-de-Tabajo-PDF.pdf

Molinero, A. y. (2003). Transporte Público: Planeación, diseño, operación y administración. Mexico: Limusa.

MTOP. (2010). Manual para estudios de origen y destino de transporte de pasajeros y mixto en áreas municipales distritales y metropolitanas. Quito: Registro Oficial.

UNE, Norma. (2006). Ministerio de Fomento España. Norma Europea. Obtenido de Manual de Apoyo para la Implantación de la Gestión de la calidad según Norma UNE-EN 13816: https://www.fomento.gob.es/recursos_mfom/pdf/6336A7393BBF-426C-AD69-

1F3C59833A19/25583/Cap1ElmodelodecalidadsegunUNEEN13817.pdf Unidad de Movilidad Latacunga. (2019). Compañias de transporte público Latacunga. Latacunga.

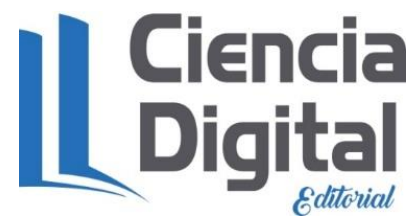


Para citar el artículo indexado.

Aguilar G. \& Luis J. (2019). Análisis de la metodología de la ANT para fijar la tarifa de transporte modalidad taxi., Revista electrónica Ciencia Digital 3(2), 5-25. Recuperado desde:

http://www.cienciadigital.org/revistascienciadigital/index.php/VisionarioDigital/article/view/391/ $\underline{875}$

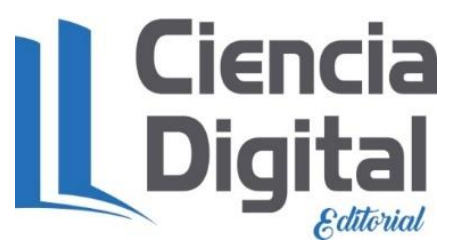

El artículo que se publica es de exclusiva responsabilidad de los autores y no necesariamente reflejan el pensamiento de la Revista Ciencia Digital.

El artículo queda en propiedad de la revista y, por tanto, su publicación parcial y/o total en otro medio tiene que ser autorizado por el director de la Revista Ciencia Digital.
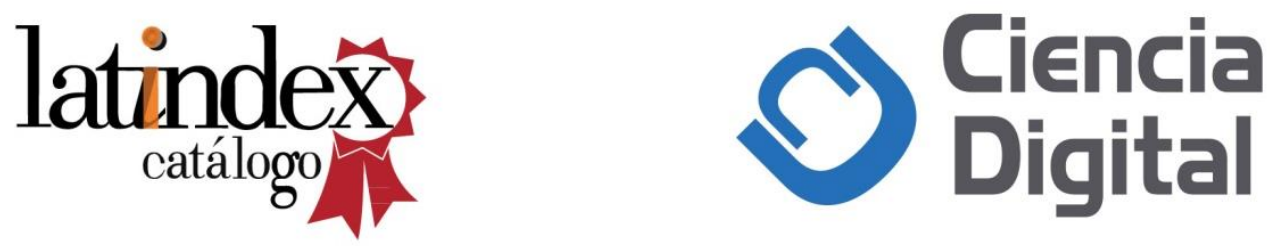